\title{
Medición de la Integralidad Educativa. Una Aproximación desde los Nuevos Indicadores de Calidad Escolar
}

\author{
Measuring the Integrality of Education. \\ An Approach from the New Indicators of School Quality
}

\author{
Víctor Castillo Riquelme1* y Juan Escalona Bustos ${ }^{2}$ \\ ${ }^{1}$ Universidad del Bío-Bío e ${ }^{2}$ Investigador independiente de políticas sociales
}

\begin{abstract}
En esta reflexión teórica se expone un conjunto de nuevos indicadores para medir calidad escolar incorporados recientemente por el SIMCE al proceso de medición de la calidad de los establecimientos educativos de enseñanza básica y media en Chile. Cada indicador se describe y justifica a la luz de la evidencia empírica acumulada por investigaciones previas, a la vez que se emiten juicios críticos y orientaciones acerca de su interpretación. Se sostiene que estos nuevos indicadores constituyen un avance importante en la medición de la calidad vista desde un enfoque de integralidad, por lo que ayudarían a estrechar la brecha entre lo que se espera de los establecimientos y lo que se mide de ellos. No obstante, es posible identificar algunos elementos susceptibles de mejora que ameritan una revisión seria por parte de la política educativa a fin de mejorar la integralidad de la educación y su expresión métrica.
\end{abstract}

Palabras clave: Integralidad de la educación, Calidad de la educación, Evaluación estandarizada, Política educativa, Medición educativa.

This theoretical review shows a set of new indicators to measure school quality recently incorporated by SIMCE to the process of measuring the quality of educational establishments of primary and secondary education in Chile. Each indicator is described and justified in the light of empirical evidence accumulated by previous researches, while critical judgments and guidance on its interpretation are issued. It is argued that these new indicators are an important step forward in quality measurement, view from an approach of integration, which would help narrow the gap between what is expected of establishments and measured them. However, it is possible to identify some elements for improvement that merit serious review by the education policy to improve the integrity of education and its metric expression.

Keywords: Integrality of education, Quality of education, Standardized assessment, Educational policy, Educational measurement.

*Contacto: vcastillo@ubiobio.cl

issn: 1989-0397

www.rinace.net/riee/

https://revistas.uam.es/riee
Recibido: 29 de abril de 2016

$1^{\text {a }}$ Evaluación: 20 de julio de 2016

Aceptado: 9 de agosto de 2016 


\section{Introducción}

La comunidad académica y los actores sociales discuten si los resultados en pruebas estandarizadas son una imagen confiable y válida de lo que sucede en el aula y a la vez se preguntan si acaso las propuestas formativas centradas en la evaluación y en la rendición de cuentas son el camino más adecuado para superar la estabilidad de los resultados escolares y conducir los aprendizajes hacia un estándar de calidad más alto e integral.

En medio de las tensiones y resistencias que los sistemas de accountability (rendición de cuentas) suelen suscitar (McMeekin, 2006), la Agencia de Calidad de la Educación, dependiente del Ministerio de Educación de Chile, ha incorporado al proceso de evaluación de los establecimientos una nueva política denominada Otros Indicadores de Calidad Escolar (OIC), la cual busca promover el desarrollo socio-personal de los estudiantes. Estos nuevos indicadores ampliarían el concepto de calidad, reconociendo que la educación formal no solo se hace cargo del entrenamiento curricular, sino que además le es atingente la promoción de las habilidades no curriculares de los estudiantes. Según Heckman, Stixrud y Urzua (2006), para un amplio rango de conductas sociales y outcomes (resultados) económicos las variables socio-personales son tanto o más importantes que las de mayor exigencia cognitiva.

Algunos de los OIC tienen un rol instrumental para la mejora de los aprendizajes disciplinares, pero a la vez constituyen fines que le son propios a la educación entendida como formación humana y social, es decir, expresada en el logro de hábitos de ser, de hacer, de pensar, de sentir y de querer (Daros, 2012).

Este artículo tiene por objetivo ofrecer una reflexión en torno a la incorporación de los OIC a la política educativa nacional y su eventual aporte a la integralidad de la educación. El primer apartado describe al enfoque de integralidad que se ha ido instalando en la retórica académica, mientras que el segundo describe la medición educacional vista desde los avances y desafíos del Sistema de Medición de la Calidad de la Educación (SIMCE) vigente en Chile. El tercer apartado, y núcleo de este artículo, presenta cada uno de los OIC a la luz de las definiciones que la misma Agencia de Calidad de la Educación explicita, y se debate en torno a ellos. Este análisis es construido sobre la base de la revisión de la literatura tanto a nivel empírico como teórico. Finalmente se incorporan conclusiones y sugerencias de política educativa para la mejora de estos nuevos indicadores.

\section{Enfoque de integralidad de la educación}

Los magros resultados así como la escandalosa inequidad que manifiesta el sistema escolar chileno han hecho de la educación una preocupación social creciente durante los últimos años. El movimiento estudiantil del año 2006, denominado Revolución Pingüina, habría marcado un punto de inflexión en la agenda educativa de los gobiernos postdictadura, visibilizando el malestar social e impactando fuertemente en la opinión pública. Con ello, la acción colectiva converge en transformación social, presionando las medidas legislativas y las acciones de política pública específicas. Como consecuencia se han orientado cambios al modo prevaleciente de entender a la educación, a la vez que se han sumado nuevos desafíos asociados al resguardo de la calidad y equidad del sistema. Tales medidas son recogidas por la Ley General de Educación (LGE) promulgada en 2009, que vino a 
derogar a la antigua Ley Orgánica Constitucional de Enseñanza (LOCE), y por el Sistema Nacional de Aseguramiento de la Calidad aprobado en 2011. De acuerdo con Bellei, Contreras y Valenzuela (2010) estas propuestas nacen en respuesta a la presión ciudadana, puesto que las transformaciones educacionales no se encontraban dentro de las prioridades de la agenda política con anterioridad al movimiento estudiantil de 2006.

Aunque las apuestas legislativas dejaron al margen algunos temas de gran relevancia o bien no lograron satisfacer las altas expectativas ciudadanas, hubo aportes significativos que, proyectados en el tiempo, encaminarían a nuestro sistema educativo hacia una institucionalidad más sistémica y acorde con las necesidades socio-personales de la población estudiantil. En este contexto una de las innovaciones conceptuales de mayor alcance en la promulgación de la LGE es la sustitución de Enseñanza por Educación, cambio en la denominación de la ley que implicaría la adopción de un enfoque centrado en el desarrollo integral de los alumnos que va más allá de los logros en los estándares de aprendizaje. Se espera así que una educación de calidad incorpore nuevas finalidades vinculadas con el respeto a los derechos humanos y las libertades fundamentales, el ejercicio de la tolerancia, de la paz y del respeto a la diversidad.

Siguiendo a León (2012), en la integralidad de la educación confluye la formación tanto de la mente como del cuerpo, incorporando la cognición, la emocionalidad, la afectividad, la ciudadanía social y la vida productiva laboral. Sin embargo, esta concepción de la integralidad no es reciente. Ya en el año 1996 el informe Delors, promocionado por la UNESCO, puso de manifiesto los cuatro pilares sobre los que debería cimentarse la educación en el siglo XXI en un marco de pluridimensionalidad: Aprender a Conocer, Aprender a Hacer, Aprender a Vivir Juntos y Aprender a Ser, categorías que de acuerdo con los autores debiesen recibir una atención equivalente en la formulación de las políticas educativas, aun cuando estas no se desarrollan en paralelo sino que convergen en una educación holística e interactiva. El Aprender a Conocer implica, por una parte, dotar a los estudiantes de una cultura general lo suficientemente vasta para orientar la comprensión de la realidad y, por otra, el desarrollo en profundidad de un número limitado de materias en consonancia con los intereses diversos del estudiantado. El Aprender a Hacer consiste en un saber de tipo procedimental que pone en práctica los aprendizajes en pos de la formación para el mercado del trabajo, instalando competencias que permitan al individuo trabajar en equipo, asumir riesgos y fortalecer su capacidad de iniciativa. Por su parte, en un mundo cada vez más competitivo, el Aprender a Convivir aporta mediante el ejercicio de la empatía al descubrimiento del otro, al reconocimiento de la diversidad e interdependencia humana, a la participación de proyectos comunes y a la resolución o gestión de los conflictos. Elementos que se traducen en una convivencia nutritiva para el funcionamiento de las sociedades. Finalmente, el Aprender a Ser refiere a conferir la libertad de pensamiento, de juicio, de sentimientos y de imaginación para alcanzar la plenitud y autonomía individual. Ello involucra por lo demás la autorrealización estética, artística, deportiva, científica, cultural y social.

La demanda por una educación integral de calidad ha suscitado una mayor preocupación por las esferas de enseñanza no formal e informal, situación que se tradujo en el reconocimiento oficial de tales campos en la LGE (2009). En efecto, esta ley define a la enseñanza no formal como un proceso formativo sistemático, al margen de la obtención de un título o nivel de estudio, incorporando a este campo las actividades socioeducativas, voluntariados y capacitaciones laborales de diversa índole. La educación Informal por su 
parte es definida como un proceso no estructurado de asimilación de experiencias dadas por la interacción del individuo con su entorno y sin la tuición del establecimiento educacional.

La taxonomía anterior no da cuenta de la yuxtaposición que tienen estos conceptos en la práctica educativa, haciéndose cada vez más habitual encontrar, al interior de las aulas, actividades asociadas con aprendizajes orientados al desarrollo socio-personal de los estudiantes, de forma que la educación no formal se entremezcla con el quehacer pedagógico tradicional. Colom (2005) advierte que la delimitación entre educación formal y educación no formal estaría determinada más bien por el carácter jurídico que le conceden las leyes educativas que por el carácter pedagógico de las mismas. Así pues, contenidos otrora exclusivos de la educación no formal, tales como conciencia socioambiental, ciudadanía, equidad de género, hábitos de vida saludable y convivencia social se estarían incorporando al acervo escolar formal (Colom, 2005).

En Chile, a partir del proceso de modernización de la educación a mediados de los noventa y bajo la influencia del citado informe Delors de la UNESCO, las finalidades de la educación se amplían, reestructurando el marco curricular, principalmente por medio de la oficialización de los denominados Objetivos Fundamentales Transversales. Estos objetivos, eventualmente, vienen a formalizar los aprendizajes de carácter general asociados a los cuatro pilares de la educación, a fin de potenciar el desarrollo de los estudiantes en el plano intelectual, moral, social y personal, complementando así a los Objetivos Fundamentales Verticales vinculados a parcelas específicas del conocimiento.

Con todo, este nuevo escenario, configurado sobre la base de la retorica de la integralidad, ha instalado progresivamente en las instituciones escolares una mayor demanda formativa, responsabilizándola ya no sólo de la transmisión del conocimiento formal, sino que también de la entrega de valores (García y Mínguez, 2011), formación ciudadana (García-Huidobro, Ferrada y Gil, 2014), habilidades blandas, educación para la salud (Salinas, Correa y Vio 2013), y en definitiva un desarrollo socio-personal armonioso y consecuente con el porvenir de una sociedad más avanzada.

Si bien los Objetivos Fundamentales Transversales, la permeabilidad del sistema escolar a los contenidos otrora no formalizados, así como las nuevas tendencias que llegan de la mano de una nueva ley general de educación viabilizan una calidad educativa multidimensional, esta entraría en tensión al considerar un sistema de medición de calidad opaco que otorga una importancia exacerbada a la transmisión del conocimiento tradicional.

\section{Medición de la calidad educativa en Chile}

Desde la creación del Sistema de Medición de la Calidad de la Educación (SIMCE) en 1988, las decisiones de política educativa en Chile se han alimentado en gran parte por los resultados de pruebas estandarizadas en distintas áreas de aprendizaje. En la adopción de este enfoque basado en estándares subyace la idea de que la medición y recolección de información relativa a los logros de aprendizaje curricular y su uso posterior para la toma de decisiones contribuiría al mejoramiento de la calidad y equidad de la educación, objetivo explícito del SIMCE del cual existe una profunda controversia acerca de su grado de alcance. 
Si bien la pretensión de cuantificar los resultados escolares había estado presente en experiencias anteriores al SIMCE, tales como la Prueba Nacional (1968-1971) y el programa PER (Programa de Evaluación del Rendimiento Escolar) (1982), ninguna de estas logró prolongarse en el tiempo de forma sostenida, ni mucho menos impactar en la comunidad educativa como lo ha hecho el SIMCE hasta el presente. En tal sentido, a casi treinta años de su implementación, este sistema ha aplicado de forma ininterrumpida evaluaciones en los subsectores de Matemáticas y Lenguaje, ha incorporado nuevas ramas del conocimiento, ha extendido la cobertura de las pruebas a más etapas de la vida escolar e inclusive ha diseñado un formato para evaluar el aprendizaje en estudiantes con discapacidad sensorial. Todas estas innovaciones han posicionado al SIMCE como un referente informativo del progreso curricular de los establecimientos, instalándolo profundamente en la tradición del sistema educativo chileno.

Pese a lo anterior, en un escenario caracterizado por la baja calidad y equidad de la educación, las críticas que apuntan a la validez del SIMCE se han multiplicado en los últimos años. Hay quienes afirman que los sistemas de evaluación nacionales basados en pruebas estandarizadas adolecen de parcelar la calidad educativa, restringiéndola al desempeño en áreas curriculares claves (Murillo y Román, 2010). Ello implicaría por lo demás una despreocupación por las asignaturas no medidas tales como Filosofía, Artes y Música, las que eventualmente se extinguirían del marco curricular (Revista Docencia, 2009). Si bien este sesgo se ha ido atenuando en la medida que se han añadido más subsectores a las evaluaciones, estas siguen dentro del espacio curricular, por lo que una educación pensada desde ámbitos como la convivencia social, la ética y la praxis queda marginada del estándar de calidad.

En línea con la crítica a la parcelación educativa, Casassus (2010) añade que la definición de los estándares de contenido es una actividad de naturaleza política-subjetiva y, por tanto, sujeta al poder de decisión de los tecno-burócratas. El reduccionismo evaluativo que se atribuye al uso de pruebas de rendimiento no se condice con las nuevas tendencias en educación que a nivel retórico conceptualizan la calidad desde una visión holística y multidimensional, situación que rompe con el isomorfismo establecido entre el constructo y su medición. Parafraseando a Murillo y Román (2010), existe una presión creciente hacia los establecimientos educativos por entregar una educación integral posibilitadora del desarrollo pleno de las potencialidades del individuo que no se traduce en sistemas de monitoreo escolar igualmente integrales.

Con todo, estos cuestionamientos en torno a la validez del SIMCE no pueden ser pasados por alto, no tan sólo por la legitimidad de enunciar una crítica, sino que también por tratarse de un sistema de altas consecuencias para los distintos agentes involucrados. Por una parte, el SIMCE tiene un uso instrumental en el diseño de intervenciones diferenciadas y en la asignación de recursos. En la medida que el SIMCE solo proporciona una imagen parcial de la realidad objetivo, las decisiones de política pública eventualmente reducirían su racionalidad a la vez que incrementarían los sesgos de focalización. Por otra parte, a este sistema de medición se le atribuyen ciertos incentivos perversos, al que tributan por ejemplo los procesos de selección y descreme de los alumnos por parte de los establecimientos educativos como garantía de un buen desempeño en las pruebas de rendimiento (Ortiz, 2012), así como la estigmatización de las escuelas más vulnerables, muchas veces alimentada por la prensa, a partir de procesos de Ordenación, Jerarquización o Semaforización de los resultados escolares (Budnik et al., 2011). 
Frente a todo lo anterior, un avance reciente ha sido la introducción de los denominados Otros Indicadores de Calidad Escolar (OIC), los cuales entregan información sobre aspectos relacionados con el desarrollo personal y social de los estudiantes. Si bien en la literatura académica el concepto socio-personal aparece bajo denominaciones y marcos teóricos diversos, como lo son las teorías de inteligencias múltiples e inteligencia emocional, existe consenso en destacar el aporte que genera al relevar la importancia de las necesidades del individuo en tanto a persona y en tanto a miembro de la sociedad, dentro de un sistema que por tradición ha estado más preocupado por las destrezas intelectuales y curriculares del alumnado (Goñi y Zarandona, 1998). Del mismo modo existe consenso en destacar la naturaleza multidimensional del concepto, aun cuando los contenidos de lo socio-personal sean un permanente objeto de discrepancias y debate, razón por cual la cobertura de este concepto dependerá en gran parte de su operacionalización en el marco de las políticas educativas, para este caso la política de los OIC. En los OIC se agrupan ocho indicadores: Autoestima y Motivación Escolar, Convivencia Escolar, Participación y Formación Ciudadana, Hábitos de Vida Saludable, Equidad de Género, Asistencia Escolar, Retención Escolar y Titulación Técnico Profesional. En su totalidad, los OIC junto con los estándares de desempeño tradicionales tributarían a una medición Multidimensional de la Calidad Escolar, estrechando la brecha entre lo que conceptualmente se entiende por calidad y su operacionalización evaluativa.

\section{Presentación de los Otros Indicadores de Calidad}

Si bien los OIC fueron incorporados por primera vez a los cuestionarios de contexto en el año 2013, estos mismos cuestionarios ya con anterioridad exploraban algunos atributos similares. Por consiguiente, la innovación más importante no descansa en la ampliación de los contenidos medidos por el SIMCE, sino más bien en la asignación de un peso significativo a los atributos de desarrollo socio-personal en el proceso de Ordenación y Evaluación de los establecimientos. Esto es así porque la lógica con la que operan las reformas basadas en estándares descansa sobre la base de principios conductistas del aprendizaje (Casassus, 2010), de forma que si una medición no genera consecuencias en los actores comprometidos sería poco probable que estos cambiaran su conducta en el rasgo medido, con los consiguientes costes individuales, institucionales y sistémicos. En este sentido, si los OIC no tienen un peso relativo en las políticas de evaluación y asignación de recursos, los esfuerzos directivos y pedagógicos estarán centrados en mejorar los puntajes de sus alumnos en las pruebas estandarizadas SIMCE, ignorando eventualmente las potencialidades no académicas de sus estudiantes. En efecto, esta ha sido una crítica constante hacia el sistema SIMCE.

La política basal de la evaluación de los establecimientos educativos se denomina Ordenación, la cual consiste en rankear (ordenar de acuerdo a una puntuación) y luego clasificar a las unidades educativas en cuatro categorías de desempeño: Insuficiente, Medio-Bajo, Medio y Alto (ACE, 2014). Este proceso de Ordenación instala consecuencias en los establecimientos categorizados como Insuficientes que pueden inclusive llevar al cierre de los mismos. Como señala Meckes et al. (2015), gran parte de las críticas al sistema SIMCE competen en realidad a este tipo de políticas más que al proceso de medición. 
La ponderación de los distintos componentes de la calidad educativa se distribuye para efectos de la Ordenación en un $67 \%$ para los estándares de aprendizaje ${ }^{1}$ y el $33 \%$ restante para los indicadores de progreso SIMCE, puntaje SIMCE y los ocho OIC, fraccionándose este porcentaje en partes iguales. Cuando todos los OIC son calculables, el porcentaje global de estos en la política de Ordenación es de un 26,4\% y el peso de cada OIC es de un 3,3\%. En los establecimientos en donde no procede medir el OIC de Equidad de Género y/o el OIC de Titulación Técnico Profesional, el porcentaje global y específico de los OIC en la política de Ordenación se alteran levemente.

La experiencia previa ha mostrado que cuando se comparan establecimientos de distinta composición socioeconómica se pone en riesgo estigmatizar a los establecimientos más vulnerables, creando la ilusión de que establecimientos con mejores resultados SIMCE garantizan una mayor calidad. Para evitar ello, todos los indicadores de calidad son calculados en grupos comparables, es decir, relativamente homogéneos en cuanto a condiciones de educabilidad.

\subsection{Hábitos de Vida Saludable}

El indicador Hábitos de Vida Saludable evalúa las actitudes y conductas autodeclaradas de los estudiantes en relación con la vida saludable, y también sus percepciones sobre el grado en que el establecimiento promueve hábitos beneficiosos para la salud. Las subdimensiones de este indicador son las siguientes: Hábitos alimenticios, Hábitos de vida activa y Hábitos de autocuidado. (ACE, 2014, p.1)

Los cambios en el perfil epidemiológico de la población escolar en Chile han hecho de los Hábitos de Vida Saludable una preocupación de creciente interés para las políticas educativas y de salud pública. Se espera mediante la educación para la salud promover hábitos que beneficien el bienestar físico, mental y social de los estudiantes y a la vez reducir las conductas de riesgo, particularmente aquellas vinculadas con la etiología y progresión de enfermedades crónicas no transmisibles. Para medir las conductas y actitudes frente a estos hábitos, el indicador de Hábitos de Vida Saludable contempla tres dimensiones: Hábitos Alimenticios, Hábitos de Vida Activa y Hábitos de Autocuidado.

Los ítems de la dimensión Hábitos Alimenticios exploran la frecuencia de consumo de distintos alimentos, así como cuestiones vinculadas a las actividades de promoción que el establecimiento realiza a este respecto. Es decir, mide no solo el resultado o fin perseguido (alimentación saludable), sino que también los medios con los que el establecimiento busca impactar sobre él. La decisión política de ponderar estos esfuerzos, desde una perspectiva pragmática, puede no ser acertada y más bien su inclusión parece estar justificada por la necesidad de monitorear el hecho de que, independientemente del impacto, las escuelas den su mayor esfuerzo por mejorar la calidad integral de sus alumnos. Por lo demás, los factores que pesquisa la dimensión Hábitos Alimenticios están avalados por un robusto cuerpo teórico y empírico.

La dimensión Hábitos de Vida Activa se compone de ítems que permiten cuantificar tanto la participación de los estudiantes en actividades físicas como las acciones de promoción por parte del establecimiento escolar. Dimensión que entra en directa sintonía con la

\footnotetext{
${ }^{1}$ Los estándares de aprendizaje miden a través de las pruebas SIMCE el dominio del currículum nacional de los estudiantes con base a la clasificación del establecimiento en tres niveles de logro: Adecuado, Elemental e Insuficiente.
} 
recomendación de la OMS de realizar al menos 60 minutos de actividad física diaria. Un acierto importante es que se pregunta por la participación en actividad física tanto en la asignatura de Educación Física como en otras instancias al interior de la escuela, revitalizando la importancia de las Actividades Curriculares de Libre Elección (ACLES) vinculadas a los deportes (Basquetbol, Fútbol, Tenis, etc.) y a otros talleres de exigencia física (Baile, Cheerleaders, Excursión, etc.). Estas actividades no solo tienen una valoración como medios contra el sedentarismo, sino que también se ajustan a uno de los principios básicos de la integralidad educativa, este es, que los alumnos puedan desarrollar sus capacidades al máximo atendiendo a sus diversos intereses.

La dimensión Hábitos de Autocuidado explora, bajo la premisa teórica de que las personas adquirimos responsabilidad de nuestra salud a través del aprendizaje, conductas vinculadas a la sexualidad, la higiene y el consumo de drogas lícitas e ilícitas. Cada tópico está avalado por su relevancia en el desarrollo mental, social y físico de los estudiantes, así como por su aporte a la reducción de costos sociales asociados. Si bien la incorporación de estos temas era, en efecto, una necesidad sentida al momento de discutir acerca de la calidad de los establecimientos, los tópicos de autocuidado por sí solos ameritan una pesquisa más amplia e independiente. En efecto, de acuerdo con los cuestionarios aplicados en la última medición, cada tópico de la dimensión autocuidado fue abordado por tres ítems cada uno. Exploración que no se condice con la multidimensionalidad que subyace en variables de esta naturaleza.

\subsection{Participación y Formación Ciudadana}

La Participación y formación ciudadana se relaciona con las percepciones y actitudes de estudiantes, padres y apoderados sobre el grado en que la institución fomenta la participación, el compromiso de los miembros de la comunidad educativa y la vida democrática. El indicador contempla las siguientes subdimensiones: Sentido de pertenencia, Participación y Vida democrática. (ACE, 2014, p.1)

Una educación pensada desde la Participación y la Formación Ciudadana constituye la piedra basal sobre la que ha de cimentarse una democracia exitosa, no tan solo debido a la necesidad de contar con un electorado educado capaz de expresar sus intereses y actuar de manera colectiva (NDI, 2011), sino que también por propiciar actitudes y conductas vinculadas con el pluralismo, la cohesión, la solidaridad, la tolerancia y el ejercicio de la paz. Dada esta apremiante necesidad, la Participación y la Formación Ciudadana se han constituido en indicadores de relevancia al juzgar la calidad de los establecimientos educativos.

La dimensión Sentido de Pertenencia explora en qué medida los estudiantes comparten los valores, preferencias, ideologías y logros que el establecimiento educativo persigue (Redon, 2010); sin embargo, puede no visibilizar al fenómeno de polarización social, esto es, cuando los grupos sociales se sienten identificados con miembros de su propio conjunto, pero distantes respecto de otros (Gasparini y Molina, 2006), situación particularmente relevante en sociedades fragmentadas como la latinoamericana. Es decir, la dimensión Sentido de Pertenencia, aunque es importante, no es garantía suficiente para asegurar la cohesión. Esta última requiere ser explorada a partir de una dimensión de mixtura social que integre la diversidad sociocultural como criterio de calidad de los establecimientos escolares. La mixtura social genera efectos positivos en el rendimiento escolar de los estudiantes de bajo nivel socioeconómico (efecto par) (Ortiz, 2015), y además consolida principios básicos para el funcionamiento democrático al proveer significados 
compartidos sobre los cuales se forjará el sentido de comunidad propio del ámbito de lo público (Peña, 2007).

En su origen etimológico, participar significa tomar parte de algo, habitualmente una cuota de poder en el proceso decisorio (Cohen y Franco, 1992). Con base en ello, la dimensión de Participación debiera dar cuenta de los distintos grados de intensidad con que los apoderados y alumnos se hacen protagonistas legítimos de las decisiones que les afectan como comunidad educativa. No obstante, los ítems que exploran esta dimensión más bien homologan la participación a la asistencia de los apoderados y alumnos a diversos espacios de encuentro, sin que ello implique necesariamente un involucramiento influyente en las decisiones finales de la comunidad educativa. Más bien, la medición se orienta al nivel informativo y consultivo de la participación. Entre otras instancias, el indicador considera la realización de actividades extraescolares como presentaciones, kermeses, bingos, eventos solidarios e inicio y cierre de periodos académicos. En consecuencia, la pesquisa de esta dimensión no visibilizaría, tal vez, los componentes más importantes de la participación, vale decir, el control ciudadano y la codecisión por parte de los escolares, quienes amparados en la convención internacional de derechos de los niños, niñas y adolescentes, exigen con legitimidad que sus opiniones sean escuchadas y tomadas en cuenta.

En Chile la democracia ha sido conceptualizada como una deuda por cubrir (Paredes, 2011) que ha operado sobre una sociedad despolitizada (Mayol, Araya, Azócar y Azócar, 2011), donde la capacidad efectiva de ejercer los derechos ciudadanos ha sido limitada por la resistencia de enclaves autoritarios heredados de la dictadura que no han sido resueltos por los gobiernos posteriores (Garretón y Garretón, 2010). Problemáticas que también le son extensivas a la Vida Democracia intra-escuela, donde un conjunto de vicios y resabios afectan al funcionamiento de los Centros de Alumnos, Consejos de Curso, así como a la promoción docente de la actividad democrática. Los ítems de esta dimensión indagan precisamente el grado en que los establecimientos favorecen las buenas prácticas democráticas, informando acerca de los niveles de participación electoral con que se escogen los representantes escolares, la legitimidad de los mismos y la promoción de actitudes básicas para ser un ciudadano democrático.

\subsection{Clima de Convivencia Escolar}

"El indicador Clima de convivencia escolar considera las percepciones y las actitudes que tienen los estudiantes, docentes y padres y apoderados con respecto a la presencia de tres subdimensiones: Ambiente de respeto, Ambiente organizado y Ambiente seguro" (ACE, 2014, p. 2).

Un buen Clima de Convivencia Escolar es naturalmente un objetivo obligatorio para cualquier sistema escolar. Según el informe Delors (1996) el aprender a vivir juntos, en el contexto escolar, implica el descubrimiento gradual del otro y la participación de proyectos comunes como el método más eficaz para evitar o resolver pacíficamente los conflictos. La Convivencia Escolar es a la vez el factor OIC que explica la mayor varianza de los resultados académicos de los alumnos (ACE, 2014), debido a que un buen clima escolar trae aparejadas condiciones de trabajo que permiten a los docentes transitar por más contenidos, abordarlos en mayor profundidad y prestar más atención a las necesidades individuales, impactando directamente en el aprendizaje y bienestar de los estudiantes (Arancibia, 2004). Con un conjunto cercano a los sesenta ítems, el OIC de Clima de 
Convivencia Escolar constituye el indicador que es abordado en mayor profundidad por los cuestionarios de contexto para alumnos. La amplitud de este este indicador y sus dimensiones como instrumento de tamizaje permiten evaluar una gran cantidad de variables vinculadas con la convivencia.

Los ítems de la dimensión Ambiente Seguro exploran la violencia escolar en sus múltiples manifestaciones, así como la percepción de seguridad en distintos espacios del establecimiento escolar. Gran relevancia cobra en esta dimensión el fenómeno del bullying como expresión de la violencia física, psicológica, social y tecnológica entre pares. A su vez se explora la frecuencia con que se manifiestan determinadas situaciones que atentan contra la seguridad, como por ejemplo el robo, las amenazas, las peleas y las agresiones, entre otras.

La dimensión Ambiente de Respeto mide el trato entre los distintos actores del establecimiento escolar, pesquisando que el clima se sustente en la confianza hacia los profesores y en la cooperación con el orden. También incorpora un conjunto de ítems que exploran la presencia de discriminación por distintas características personales al interior del establecimiento. Esta exploración lógicamente esconde la discriminación institucionalizada en las prácticas de selección escolar, muchas veces amparada en el verso de la diversidad de proyectos educativos que induce paradójicamente a la configuración de una población escolar homogénea.

La dimensión Ambiente Organizado mide, por una parte, la existencia y efectividad de las normas de convivencia y, por otra, las creencias acerca de la transgresión de las mismas. Se espera no solo la fijación de sanciones sino que también que los alumnos tengan actitudes negativas hacia las conductas moralmente reprochables (robar, hacer daños, copiar en las pruebas).

\subsection{Motivación Escolar y Autoestima Académica}

El indicador Autoestima académica y motivación escolar considera, por una parte, la autopercepción y la autovaloración de los estudiantes en relación con su capacidad de aprender y, por otra parte, las percepciones y actitudes que tienen los estudiantes hacia el aprendizaje y el logro académico. Las sub dimensiones de este indicador son: Autopercepción y autovaloración académica; motivación escolar. (ACE, 2014, p.1)

En el seno de la Psicología Positiva modelos teóricos han relevado la importancia de educar para la felicidad (Seligman, Ernst, Gillham, Reivich y Linkins, 2009), promoviendo el enriquecimiento integral de los alumnos y su bienestar psicoemocional (Romero y Pereira, 2011). El OIC Motivación Escolar y Autoestima Académica entra en directa sintonía con esta corriente teórica, aunque, como es evidente, el indicador es focalizado al ámbito escolar, restringiendo su potencial aporte a la consecución de objetivos pedagógicos que trascienden al ideal académico.

La dimensión de Autoestima Académica es una concepción diferente a la Autoestima Global. Mientras que la Autoestima Global se vincula fuertemente con los indicadores de bienestar psicológico, la Autoestima Académica adquiere más relevancia como predictor del rendimiento escolar (Rosenberg, Schooler, Schoenbach y Rosenberg, 1995). Aunque sea importante que las escuelas transmitan altas expectativas de logro a sus alumnos y fomenten una percepción positiva de sí mismos en el plano académico, no se puede marginar a los otros dominios de la autoestima que también constituyen objeto de intervención socioescolar. En definitiva, que los estudiantes se sientan bien consigo 
mismos debiese ser el principio guía de este indicador en un marco de integralidad educativa. Misma situación acontece con la dimensión de Motivación Escolar.

\subsection{Asistencia Escolar}

"El indicador de Asistencia escolar evalúa la capacidad de un establecimiento para promover que sus estudiantes asistan regularmente a clases" (ACE, 2014, p. 1).

Más allá de ser el agente formal para la transmisión del conocimiento, la escuela se constituye en un espacio de convivencia simétrica, donde los niños y adolescentes adquieren habilidades sociales y una identidad personal, resultado de la interacción constante con su grupo de pares, que difícilmente podrían desarrollar en otros espacios de socialización. Ello hace de la Asistencia Escolar una variable clave para el logro de ciertos objetivos educacionales como la retención escolar, la asimilación del conocimiento curricular y la instalación de actitudes prosociales, entre otras. Los estudios a este respecto señalan que los alumnos con altas tasas de asistencia escolar alcanzan mejores resultados en las pruebas SIMCE y desarrollan pautas de comunicación e interacción más nutritivas con su grupo de referencia (ACE, 2014). Los niveles de asistencia, sobre todo en los primeros niveles de transición, también han demostrado tener efectos significativos en el rendimiento ex post.

Si bien la importancia de esta variable es indiscutida, su uso como indicador de calidad en el proceso de Ordenación de los Establecimientos puede no ser pertinente debido a que se trata de un medio más que de un fin, por lo que sus eventuales efectos en calidad educativa debiesen ser absorbidos por los otros indicadores considerados. Además, la Asistencia Escolar tiene un uso actual en la asignación de recursos vía vouchers (subsidio a la demanda), razón suficiente para atraer el interés y preocupación de sostenedores y directivos. Consignar una ponderación importante a la Asistencia Escolar en el proceso de evaluación de los establecimientos podría estimular aún más ciertas externalidades negativas ya presentes en el sistema, principalmente la adulteración de los registros de asistencia.

\subsection{Retención Escolar}

"El indicador de Retención escolar considera la capacidad que tiene un establecimiento educacional para lograr la permanencia de sus estudiantes en el sistema de educación formal” (ACE, 2014, p. 2).

$\mathrm{El}$ aumento de la cobertura a cifras cercanas a la universalidad junto con el establecimiento de la obligatoriedad de más años de escolaridad trae aparejada la necesidad de retener a una gran masa estudiantil que se incorpora al sistema. Ello implica que la retención, aparte de ser un objetivo deseable para el desarrollo del país, es también una medida del éxito del sistema educativo. Por consecuencia, la inclusión de este indicador es pertinente, sobre todo en contextos educativos complejos, donde se concentra la mayor proporción de desertores 2 . Por el contrario, este indicador carecería de capacidad de discriminación en establecimientos particulares pagados o grupos socioeconómicos altos. Proyectado en el tiempo, este indicador difícilmente será sostenible en la medida que la tendencia a la

\footnotetext{
${ }^{2}$ La deserción corresponde al complemento natural de la retención.
} 
retención alcanza porcentajes cada vez más cercanos al 100\%, aunque estas cifras pueden variar de acuerdo al enfoque métrico adoptado (Mineduc, 2013).

\subsection{Equidad de Género}

El indicador Equidad de género evalúa el logro equitativo de resultados de aprendizaje obtenidos por hombres y mujeres en establecimientos mixtos. La equidad de género en el ámbito escolar, se traduce en que se transmiten expectativas similares a hombres y a mujeres respecto de su rendimiento y su futuro académico y laboral. (ACE, 2014, p.2)

La equidad de género se ha constituido en una demanda social de creciente importancia en la formulación de políticas públicas. La reciente creación del Ministerio de la Mujer y Equidad de Género, así como un conjunto de iniciativas transversalmente instaladas en programas sociales sectoriales, son prueba de un esfuerzo cada vez mayor por conseguir niveles aceptables de justicia e igualdad de oportunidades entre hombres y mujeres. El OIC de Equidad de Género no escapa de esta tendencia y busca constituirse en un parámetro de calidad del sistema educativo. Sin embargo, este indicador está centrado solo en la equiparación del logro académico sin atender a las eventuales asimetrías sociales y formativas que se expresan en las otras variables de resultados. La equiparación de puntajes de los indicadores de desarrollo socio personal, en función del sexo, debiesen adquirir una importancia similar a la asignada a la equidad de puntajes en las pruebas Simce, en virtud que la literatura informa de una historia de segmentación de género instalada más allá del plano curricular. En este sentido, el prejuicio, la socialización diferencial y las prácticas discriminatorias en la construcción valórica, sexual y ciudadanapolítica del alumnado siguen constituyendo factores no indagados por el OIC de Equidad de Género. Por lo demás, este indicador no incluye percepciones de los estudiantes que den cuenta del esfuerzo pedagógico e institucional de transmitir expectativas similares a hombres y a mujeres.

\subsection{Titulación Técnico Profesional}

El indicador Titulación técnico-profesional evalúa la proporción de estudiantes que recibe el título de técnico nivel medio, luego de haber egresado de la educación media técnicoprofesional. Este indicador no contempla a aquellos estudiantes que, habiendo recibido una educación media técnico-profesional, no realizan la práctica debido a que inician estudios de educación superior. (ACE, 2014, p.1)

Este indicador expresa de forma unidimensional y aproximada la capacidad con que la formación Técnica Profesional cumple su objetivo de vincular a los estudiantes con el mercado del trabajo. En primer término, es unidimensional, por cuanto en su construcción no convergen constructos que requieran más de una variable para su medición, lo que implica, para efectos prácticos, que la eficiencia de la formación técnica profesional es reducida a su expresión más simple, la titulación, ignorando así elementos tan importantes como la percepción de los alumnos y apoderados respecto de las acciones y gestiones que genera el establecimiento con su entorno productivo. En segundo término, es aproximado en virtud que la certificación del título técnico no es garantía de la vinculación efectiva de los estudiantes con el mercado del trabajo. Por último, y tal vez lo más importante, aun asumiendo que un alto porcentaje de egresados se integra al mercado del trabajo, poco se logra advertir respecto del dominio adquirido por los estudiantes en los contenidos de especialidad, razón por la cual la calidad de la enseñanza técnico profesional sigue siendo una caja negra para las políticas educativas. 


\section{Conclusiones}

Hoy en día la forma de medir la calidad de un establecimiento se ha ajustado a exigencias más estrictas que ponen de relieve la importancia de las variables socio-personales de su alumnado, tales como la convivencia, la autoestima, la participación y los hábitos de vida saludable, entre otras. El fortalecimiento de estas variables no solo es una condición sine qua non para mejorar el rendimiento escolar, sino que adquiere una consistencia propia que le es independiente a su rol instrumental dentro de los fines generales de la educación.

Definir operacionalmente el desarrollo socio personal es lógicamente una tarea compleja que implica en el proceso de medición seleccionar un set muy reducido de dimensiones. $\mathrm{Si}$ bien algunos atributos de interés en el plano formativo como la sustentabilidad medioambiental, el desarrollo artístico, o la interculturalidad no han sido ponderados hasta ahora como factores de la calidad educativa, es claro que los esfuerzos en materia de evaluación han significado un gran avance respecto de la otrora hegemonía de las pruebas de rendimiento SIMCE. Este antecedente da cuenta de la transición a un incipiente paradigma centrado en la calidad multidimensional de la educación y el desarrollo integral del estudiantado.

$\mathrm{Al}$ ampliar la mirada de la educación se diluye en parte la responsabilización restrictiva de los docentes por los aprendizajes de sus alumnos, toda vez que los nuevos indicadores de calidad emplazan al rol de los diversos actores que integran a la comunidad educativa, en especial, a las redes institucionales de apoyo que han visto en la escuela una población cautiva y un terreno fértil para el desarrollo de sus actividades de promoción e intervención. Los objetivos de instituciones como Senda, Sename, Junaeb, así como Centros de Salud Familiar y/o Comunitaria sintonizan directamente con el logro de mejores resultados en los indicadores de desarrollo socio-personal. Las intervenciones de carácter profesional de los asistentes de la educación: psicólogos, psicopedagogos, trabajadores sociales y orientadores, a través de sus talleres y programas, debiesen del mismo modo expresarse en mejores puntajes OIC.

Este incipiente paradigma de calidad multidimensional ha tenido entre sus bondades reducir significativamente la brecha entre lo que se exige a los establecimientos y lo que se mide de ellos, pero a la vez instala nuevas tensiones, esta vez vinculadas con el funcionamiento del sistema de accountability y las consecuencias del uso de los OIC como instrumento de control en la política educativa. Se considera relevante equilibrar el rol de las mediciones estandarizadas como instrumentos de rendición de cuentas con su potencial formativo.

La política de los OIC, si bien se encuentra en una fase inicial, puede ser mejorada si es definida con mayor claridad tanto en los objetivos que busca como en la selección y operacionalización de sus dimensiones. Resulta importante, por ejemplo, identificar la orientación de lo que se pretende medir, distinguiendo los resultados individuales que tributan más específicamente al desarrollo socio-personal, respecto de las acciones de promoción o medios con los cuales los establecimientos buscan impactar en dichos resultados. Ambas orientaciones, aunque son compatibles, refieren a objetos conceptualmente distintos, poniendo, por una parte, el foco sobre los resultados individuales y, por otro, sobre indicadores institucionales o de gestión. Además, en el conjunto de OIC es posible identificar indicadores completamente institucionales, como 
lo son los indicadores de Retención, Equidad de género, Asistencia y Titulación técnico profesional.

Los OIC y principalmente su empleo en la evaluación de los establecimientos educativos abren un amplio campo para la investigación empírica. Estudios futuros pueden evaluar el impacto de cada OIC en el proceso de Ordenación; determinar la capacidad de discriminación de los mismos; estimar los efectos de las reformas basadas en estándares sobre los resultados en variables no académicas.

Al revisar cada indicador y las dimensiones que los componen es posible identificar algunos elementos susceptibles de mejora que ameritan una revisión seria por parte de la política educativa a fin de mejorar la integralidad de la educación y su expresión métrica. Aunque la definición del qué medir será siempre una tarea de naturaleza subjetiva, es decir, vinculada a los marcos epistemológicos de quienes evalúan, la rigurosidad técnica e intelectual así como la revisión crítica, constructiva y constante pueden dar luces respecto de cómo mejorar tal proceso. Bajo esta lógica, este artículo pretende aportar las siguientes recomendaciones:

Algunos indicadores entremezclan en una misma dimensión los fines con los esfuerzos que los establecimientos realizan para conseguir los resultados deseados, situación que podría inducir a interpretaciones equívocas tanto a investigadores como a los tomadores de decisiones. A modo de ejemplo se pueden considerar los escenarios en donde los estudiantes califican de forma positiva todos los ítems vinculados con las actividades de promoción institucional pero de forma negativa los ítems asociados a los resultados.

El OIC de Autoestima Académica y Motivación Escolar requiere ser ampliado conceptualmente de modo que abarque la noción global de Autoestima y no solo su expresión académica, la cual es instrumental al paradigma centrado en el rendimiento.

El OIC de Participación y Formación Ciudadana podría incluir una dimensión vinculada con la mixtura sociocultural, es decir, la capacidad de la escuela para constituirse en un espacio de encuentro en el que participen sin distinción alumnos de origen social y cultural diverso. Además se sugiere clarificar la noción de Participación entregada por la ACE, puesto que en su medición se evoca la asistencia a actividades organizadas por el establecimiento, sin que ello implique una democratización de la toma de decisiones, siendo esta última la genuina experiencia de participación.

El indicador de Retención, pese a su importancia, puede no discriminar con eficiencia la calidad de los establecimientos educativos, sobre todo en aquellos espacios que concentran a los estudiantes de quintiles más altos. En este entendido se puede optar por metodologías estadísticas con mayor sensibilidad en la identificación de la deserción o bien por su reemplazo por otros indicadores de la integralidad educativa.

La construcción del indicador de Equidad de Género debiese proyectarse más allá de la igualación de resultados entre niños y niñas en las pruebas SIMCE, para incorporar además como criterio de calidad la igualdad de resultados en el resto de los OIC. Como complemento, y para entrar en mayor sintonía con el enfoque de integralidad, el indicador de Equidad de Género puede ser reformulado en una definición más amplia que explore la afectividad, la sexualidad y la diversidad a partir de las percepciones de los estudiantes y apoderados. 
Los cuestionarios de contexto que emplea la ACE para construir los OIC podrían incluir preguntas diferenciadas de acuerdo a la modalidad de enseñanza del establecimiento (Humanista-Científico/Técnico Profesional), a fin que la exploración del indicador de Titulación Técnico Profesional pueda responsabilizar de forma más amplia a los establecimientos por sus gestiones con el entorno productivo.

La medición y la evaluación de la calidad educativa es una tarea de naturaleza compleja y de consecuencias importantes para la comunidad educativa. Constituye una actividad que excede a lo técnico y lo procedimental, puesto que son los diferentes actores sociales los que en definitiva han puesto sus intereses en el centro del debate educativo. La reflexión y la investigación tienen el potencial para iluminar esta discusión.

\section{Referencias}

Agencia de la Calidad de la Educación. (2014). Otros indicadores de calidad educativa. Santiago de Chile: Agencia de la Calidad de la Educación. Recuperado de http://www.agenciaeducacion.cl/coordinacion-sac/cuales-son-los-otros-indicadores-decalidad/

Arancibia, V. (2004). Efectividad escolar. Un análisis comparado. Santiago de Chile: Centro de Estudios Públicos.

Bellei, C., Contreras, D. y Valenzuela, J. P. (Eds.). (2010). Ecos de la revolución pingüina. Santiago de Chile: Universidad de Chile.

Budnik, J., Cornejo, R., González, J., Redondo, J., Sánchez, E. y Sobarzo, M. (2011). La empresa educativa chilena. Educação y Sociedade, 32(115), 305-322. doi:10.1590/s010173302011000200004

Casassus, J. (2010). Las reformas basadas en estándares: Un camino equivocado. Educere et Educare, 5, 85-107.

Cohen, E. y Franco, R. (1992). Evaluación de proyectos sociales. Tres Cantos: Siglo XXI de España.

Colom, A. (2005). Continuidad y complementariedad entre la educación formal y no formal. Revista de Educación, 338, 9-22.

Daros, W. R. (2012). La educación entendida como formación humana y social. Invenio, 15(28), 1928.

Delors, J. (Comp.). (1996). La educación encierra un tesoro. Madrid: Santillana/UNESCO.

García, Á. y Mínguez, R. (2011). Los límites de la educación en valores civicos: Cuestiones y propuestas pedagógicas. Educación XXI, 14(2), 263-285. doi:10.5944/educxx 1.14.2.254

García-Huidobro, J. E., Ferrada, R. y Gil, M. (2014). La relación educación-sociedad en el discurso político- educativo de los gobiernos de la Concertación (1990-2009). Estudios Pedagógicos, 4O(1), 113-131. doi:10.4067/s0718-07052014000100007

Garretón, M. A. y Garretón, R. (2010). La democracia incompleta en Chile: La realidad tras los rankings internacionales. Revista de Ciencia Política (Santiago), 30(1), 115-148. doi:10.4067/s0718-090x2010000100007

Gasparini, L. y Molina, E. (2006). Income distribution, institutions and conflicts: An exploratory analysis for Latin America and the Caribbean. La Plata: CEDLAS. 
Goñi, A. y Zarandona, E. (1998). Desarrollo sociopersonal y educación. En A. Goñi (Ed.), Psicología de la educación sociopersonal (pp. 14-27). Madrid: Editorial Fundamentos.

Heckman, J., Stixrud, J. y Urzua, S. (2006). The effects of cognitive and noncognitive abilities on labor market outcomes and social behavior (No. 12006). Cambridge.

León, A. (2012). Los fines de la educación. Revista Científica Elctronica de Ciencias Humanas, 23, 450.

Mayol, A., Araya, J., Azócar, C. y Azócar, C. (2011). 7 fenómenos sobre educación y desigualdad en Chile. Santiago de Chile.

McMeekin, R. W. (2006). Acreditación, accountability y mejoramiento de la calidad de la educación en escuelas vulnerables. Pensamiento Educativo, 39(2), 237-253.

Meckes, L., Bustos, P., Carrasco, A., Carrasco, R., Eyzaguirre, B., García-Huidobro, J. E., ... Bravo, J. (2015). Informe Ejecutivo: Equipo de Tarea para la Revisión del Sistema Nacional de Evaluación de Aprendizajes. Recuperado de http://portales.mineduc.cl/usuarios/mineduc/doc/20150202 1034480.InformeEjecutivoE quipoTarea.pdf

Mineduc. (2013). Medición de la deserción escolar en Chile. Santiago de Chile: Autor.

Murillo, F. J. y Román, M. (2010). Retos en la evaluación de la calidad de la educación en América Latina. Revista Iberoamericana de Educación, 53(53), 97-120.

NDI. (2011). Democracia y el desafío del cambio: Una guía para aumentar la participación política de las mujeres. Washington D. C.: Autor.

Ortiz, I. (2012). En torno a la validez del Sistema de Medición de la Calidad de la Educación en Chile. Estudios Pedagógicos, 38(2), 355-373. doi:10.4067/s0718-07052012000200022

Ortiz, I. (2015). Escuelas inclusivas en el contexto de segregación social del sistema escolar chileno. Calidad en la Educación, 42, 93-122. doi:10.4067/s07 18-4565201500010004

Paredes, J. P. (2011). Ciudadanía, participación y democracia. Polis (Santiago), 10(28), 473-499. doi: $10.4067 /$ s0718-65682014000100028

Peña, C. (2007). Por qué no debemos seleccionar. En J. J. Brunner y C. Peña (Coords.), La reforma al sistema escolar: Aportes para el debate (pp. 245-252). Santiago de Chile: Universidad Diego Portales.

Redon, S. (2010). La escuela como espacio de ciudadanía. Estudios Pedagógicos, 36(2), 213-239. doi:10.4067/s0718-07052010000200013

Revista Docencia. (2009). Estandarización educativa en Chile: Un peligroso hábito. Revista Docencia, 38, 4-17.

Romero, C. y Pereira, C. (2011). El enfoque positivo de la educación: Aportaciones al desarrollo humano. Teoría de La Educación. Revista Interuniversitaria, 23(2), 69-89.

Rosenberg, M., Schooler, C., Schoenbach, C. y Rosenberg, F. (1995). Global self-esteem and specific self-esteem: Different concepts, different outcomes. American Sociological Review, 6O(February), 141-156.

Salinas, J., Correa, F. y Vio, F. (2013). Marco normativo para promover una alimentación saludable en escuelas básicas de Chile. Revista Chilena de Nutrición, 4O(3), 274-282. doi:10.4067/s071775182013000300010 
Seligman, M. E. P., Ernst, R. M., Gillham, J., Reivich, K. y Linkins, M. (2009). Positive education: Positive psychology and classroom interventions. Oxford Review of Education, 35(3), 293311. doi:10.1080/03054980902934563

\section{Breve CV de los autores}

\section{Víctor Castillo Riquelme}

Trabajador social egresado de la Universidad del Bío-Bío (Chile), actualmente estudiante del programa de magíster de Psicología Social de la Universidad de Talca (Chile) becado por la Comisión Nacional de Investigación Científica y Tecnológica (Conicyt), folio 22162591. Ha participado en diferentes proyectos de investigación y publicaciones científicas del ámbito educativo y de las ciencias sociales al alero del Centro de Investigación Educativa CIDCIE de la Universidad del Bío-Bío. E-mail: vcastillo@ubiobio.cl

\section{Juan Escalona Bustos}

Trabajador social egresado de la Universidad del Bío-Bío (Chile). Ha sido investigador colaborador del Centro de Investigación Educativa CIDCIE de la Universidad del BíoBío. Hizo su tesis de grado sobre los "Aportes socioeducativos de la integralidad de la educación: un análisis comparativo de la segmentación del desarrollo socio-personal”. 